\title{
Convergence theorem for finite family of lipschitzian demi-contractive semigroups
}

\author{
Bashir Ali and Godwin Chidi Ugwunnadi
}

* Correspondence: bashiralik@yahoo.com Department of Mathematical Sciences, Bayero University, Kano, Nigeria

\begin{abstract}
Let $E$ be a real Banach space and $K$ be a nonempty, closed, and convex subset of $E$. Let $\left\{\mathcal{J}_{i}\right\}_{i=1}^{N}$ be a finite family of Lipschitzian demi-contractive semigroups of $K$, with sequences of bounded measurable functions $L_{i}:[0, \infty) \rightarrow(0, \infty)$ and bounded functions $\lambda_{i}:[0, \infty) \rightarrow(0, \infty)$, respectively, where $\mathcal{J}_{i}:=\left\{T_{i}(t): t \geq 0\right\}, i=1,2, \ldots, N$. Strong convergence theorem for common fixed point for finite family $\left\{\mathcal{J}_{i}\right\}_{i=1}^{N}$ is proved in a real Banch space. As an application, a new convergence theorem for finite family of Lipschitzian demi-contractive maps is also proved.

Mathematics subject classification (2000) 47H09, 47J25
\end{abstract}

Keywords: Demi-contractive maps, Demi-contractive semigroup, Demicompact maps, Fixed point

\section{Introduction}

Let $E$ be a real Banach space and $E^{*}$ be the dual space of $E$. The normalized duality mapping $J: E \rightarrow 2^{E^{*}}$ is defined by, $x \in E$,

$$
J x=\left\{x^{*} \in E^{*}:\left\langle x, x^{*}\right\rangle=\|x\|\left\|x^{*}\right\|,\left\|x^{*}\right\|=\|x\|\right\},
$$

where $\langle.,$.$\rangle denotes the normalized duality pairing. For any x \in E$, an element of $J x$ is denoted by $j(x)$.

Let $K$ be a nonempty, closed and convex subset of $E$. Let $T: K \rightarrow K$ be a map, a point $x \in K$ is called a fixed point of $T$ if $T x=x$, and the set of all fixed points of $T$ is denoted by $F(T)$. The mapping $T$ is called $L$-Lipschitzian or simply Lipschitz if $\exists L>0$, such that $\|T x-T y\| \leq L\|x-y\| \forall x, y \in K$ and if $L=1$, then the map $T$ is called nonexpansive.

A one parameter family $\mathcal{J}=\{T(t): t \geq 0\}$ of self mapping of $K$ is called a nonexpansive semigroup if the following conditions are satisfied,

(i) $T(0) x=x \forall x \in K$;

(ii) $T(t+s)=T(t) \circ T(s) \forall t, s \geq 0$;

(iii) for each $x \in K$, the mapping $t \rightarrow T(t) x$ is continuos;

(iv) for $x, y \in K$ and $t \geq 0,\|T(t) x-T(t) y\| \leq\|x-y\|$.

If the family $\mathcal{J}=\{T(t): t \geq 0\}$ satisfies conditions (i) - (iii), then it is called

(a) pseudocontractive semigroup if for any $x, y \in K$, there exists $j(x-y) \in J(x-y)$ such that

$$
\langle T(t) x-T(t) y, j(x-y)\rangle \leq\|x-y\|^{2} ;
$$


(b) strictly pseudocontractive semigroup if there exists a bounded function $\lambda:[0, \infty) \rightarrow$ $(0, \infty)$ and $j(x-y) \in J(x-y)$ such that

$$
\langle T(t) x-T(t) y, j(x-y)\rangle \leq\|x-y\|^{2}-\lambda(t)\|(I-T(t)) x-(I-T(t)) y\|^{2}
$$

for all $x, y \in K$;

(c) demi-contractive semigroup if $F(T(t)) \neq \varnothing \forall t \geq 0$, there exists a bounded function $\lambda$ : $[0, \infty) \rightarrow(0, \infty)$, and $j(x-y) \in J(x-y)$ such that

$$
\langle T(t) x-q, j(x-q)\rangle \leq\|x-q\|^{2}-\lambda(t)\|x-T(t) x\|^{2}
$$

for any $x \in K$ and $q \in F(T(t))$;

(d) Lipschitzian semigroup if there is a bounded measurable function

$L:[0, \infty) \rightarrow(0, \infty)$ such that for $x, y \in K$ and $t \geq 0$,

$$
\|T(t) x-T(t) y\| \leq L(t)\|x-y\| .
$$

It is known that every strictly pseudocontractive semigroup is Lipschitzian, and every strictly pseudocontractive semigroup with fixed point is demi-contractive semi-group.

Let $E$ be a real Banach space and let $K$ be a nonempty closed convex subset of $E$. A mapping $T: K \rightarrow K$ is demicompact if for every bounded sequence $\left\{x_{n}\right\}$ in $K$ such that $\left\{x_{n}-T x_{n}\right\}$ converges, and there exists a subsequence, say $\left\{x_{n_{j}}\right\}_{\text {of }}\left\{x_{n}\right\}$ that converges strongly to some $x^{*}$ in $K . T$ is said to be demi-contractive if $F(T) \neq \varnothing$, and there exists $\lambda$ $>0$ such that $\langle T x-q, j(x-q)\rangle \leq\|x-q\|^{2}-\lambda\|x-T x\|^{2} \forall x \in K, q \in F(T)$ and $j(x-q) \in J$ $(x-q)$.

Let $T_{1}, T_{2}, \ldots, T_{N}$ be a family of self-mappings of $K$ such that $F:=\cap_{i=1}^{N} F\left(T_{i}\right) \neq \emptyset$. Then, the family is said to satisfy condition $\bar{C}$ if there exists a nondecreasing function $f$ $:[0, \infty) \rightarrow[0, \infty)$ with $f(0)=0$ and $f(r)>0 \forall r \in(0, \infty)$ such that $f(d(x, F)) \leq \| x-$ $T_{s} x \|$ for some $s$ in $\{1,2, \ldots, N\}$ and for all $x \in K$, where $d(x, F)=\inf \{\|x-q\|: q \in F\}$.

Existence theorems for family of nonexpansive mappings are proved in [1-5] and actually many others. Recently, Suzuki [6] proved the equivalence between the fixed point property for nonexpansive mappings and that of the nonexpansive semi-groups.

Both implicit and explicit, Mann, Ishikawa, and Halpern-type schemes were studied for approximation of common fixed points of family of nonexpansive semigroups and their generalizations in various spaces; see, for example [6-13], to list but a few.

In 1998, Shoiji and Takahashi [7] introduced and studied a Halpern-type scheme for common fixed point of a family of asymptotically nonexpansive semigroup in the framework of a real Hilbert space. Suzuki [8] proved that the implicit scheme defined by $x, x_{1} \in K$,

$$
x_{n}=\alpha_{n} T\left(t_{n}\right) x_{n}+\left(1-\alpha_{n}\right) x
$$

converges strongly to a common fixed point of the family of nonexpansive semigroup in a real Hilbert space. Xu [9] extended the result of Suzuki to a more general real uniformly convex Banach space having a weakly sequentially continuous duality mapping.

In 2005, Aleyner and Reich [10] proved the strong convergence of an explicit Halperntype scheme defined by $x, x_{1} \in K$,

$$
x_{n+1}=\alpha_{n} T\left(t_{n}\right) x_{n}+\left(1-\alpha_{n}\right) x
$$


to a common fixed point of the family $\{T(t): t \geq 0\}$ of nonexpansive semigroup in a reflexive Banach space with uniformly Gatéuax differentiable norm. Recently, Zhang et al. [11] introduced and studied a composite iterative scheme defined by $x, x_{1} \in K$,

$$
x_{n+1}=\alpha_{n} y_{n}+\left(1-\alpha_{n}\right) x ; y_{n}=\beta_{n} T\left(t_{n}\right) x_{n}+\left(1-\beta_{n}\right) x_{n} .
$$

Those authors proved strong convergence of the sequence $\left\{x_{n}\right\}$ to a common fixed point of the family $\{T(t): t \geq 0\}$ of nonexpansive semigroup.

Very recently, Chang et al. [12] proved a strong convergence theorem which extended and improved the results in [10,9] and some others. They proved the following theorem.

Theorem 1.1. Chang et al. [12]Let $K$ be a nonempty, closed, and convex subset of a real Banach space E: Let $\mathcal{J}:=\{T(t): t \geq 0\}$ be a Lipschitzian demi-contractive semigroup of $K$ with bounded measurable function $L:[0, \infty) \rightarrow(0, \infty)$ and bounded function $\lambda:[0, \infty) \rightarrow(0, \infty)$ such that

$$
L:=\sup _{t \geq 0}\{L(t)\}<\infty, \lambda:=\inf _{t \geq 0}\{\lambda(t)\}>0 \text { and } F:=\bigcap_{t \geq 0} F(T(t)) \neq \emptyset .
$$

Let $\left\{t_{n}\right\}$ be an increasing sequence in $[0, \infty)$ and $\left\{\alpha_{n}\right\}$ be a sequence in $(0,1)$ satisfying the following conditions,

(i) $\sum_{n=1}^{\infty}\left(1-\alpha_{n}\right)=\infty$; (ii) $\sum_{n=1}^{\infty}\left(1-\alpha_{n}\right)^{2}<\infty$. Assume that there exists a compact subset $C$ of $E$ such that $\mathrm{U}_{t \geq 0} T(t)(K) \subset C$ and for any bounded set $D \subset K$

$$
\lim _{n \rightarrow \infty} \sup _{x \in D, s \in \mathbb{R}^{+}}\left\|T\left(s+t_{n}\right) x-T\left(t_{n}\right) x\right\|=0 .
$$

Let $\left\{x_{n}\right\}$ be generated by $x_{1} \in K$,

$$
x_{n+1}=\alpha_{n} x_{n}+\left(1-\alpha_{n}\right) T\left(t_{n}\right) x_{n} .
$$

Then, the sequence $\left\{x_{n}\right\}$ converges strongly to some element in $F$.

The purpose in this article is to prove a strong convergence theorem for common fixed point for finite families $\left\{\mathcal{J}_{i}\right\}_{i=1}^{N}$ of demi-contractive semigroups in a real Banach space. As application, we also prove convergence theorem for finite family of demicontractive mappings. Our theorems generalize and improve several recent results. For instance, Theorem 1.1, which generalized, extended and improved several recent results, is a special case of our Theorem.

\section{Preliminaries}

We shall make use of the following lemmas.

Lemma 2.1. Let $E$ be a real normed linear space. Then, the following inequality holds:

$$
\|x+y\|^{2} \leq\|x\|^{2}+2\langle y, j(x+y)\rangle, \quad \forall x, y \in E \text { and } j(x+y) \in J(x+y) .
$$

Lemma 2.2. (Xu [14]) Let $\left\{a_{n}\right\}$ and $\left\{b_{n}\right\}$ be sequences of nonnegative real numbers satisfying the inequality

$$
a_{n+1} \leq\left(1+b_{n}\right) a_{n}, \quad n \geq 1 .
$$

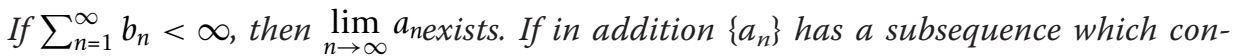
verges strongly to zero, then $\lim _{n \rightarrow \infty} a_{n}=0$. 
Lemma 2.3. (Suzuki [15]) Let $\left\{x_{n}\right\}$ and $\left\{y_{n}\right\}$ be bounded sequences in a Banach space $E$ and let $\left\{\beta_{n}\right\}$ be a sequence in $[0,1]$ with $0<\lim \inf \beta_{n} \leq \lim \sup \beta_{n}<1$. Suppose $x_{n+1}=$ $\beta_{n} y_{n}+\left(1-\beta_{n}\right) x_{n}$ for all integers $n \geq 1$ and $\lim \sup \left(|| y_{n+1}-y_{n} \|-|| x_{n+1}-x_{n}||\right) \leq 0$. Then, $\lim \left\|y_{n}-x_{n}\right\|=0$.

\section{Main Results}

Let $E$ be a real Banach space, and $K$ be a nonempty, closed convex subset of $E$. For some fixed $i \in \mathbb{N}$, let $\mathcal{J}_{i}:=\left\{T_{i}(t): t \geq 0\right\}$ be a Lipschitzian demi-contractive semigroup with bounded measurable function $L_{i}:[0, \infty) \rightarrow(0, \infty)$ and bounded function $\lambda_{i}:[0, \infty) \rightarrow(0, \infty)$ such that

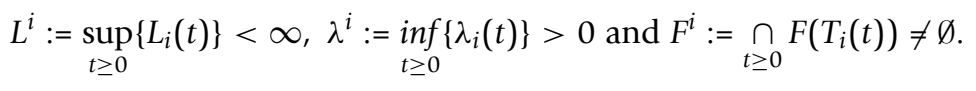

Then, for $x, y \in K, q \in F^{i}$ and $t \geq 0$,

$$
\left\langle T_{i}(t) x-q, j(x-q)\right\rangle \leq\|x-q\|^{2}-\lambda^{i}\left\|x-T_{i}(t) x\right\|^{2}
$$

and

$$
\left\|T_{i}(t) x-T_{i}(t) y\right\| \leq L^{i}\|x-y\| .
$$

Consider a family $\left\{\mathcal{J}_{i}\right\}_{i=1}^{N}$ of Lipschitzian demi-contractive semigroups of $K$ and let $L:=\max _{1 \leq i \leq N}\left\{L^{i}\right\}, L:=\max _{1 \leq i \leq N}\left\{L^{i}\right\}$ and $\lambda:=\min _{1 \leq i \leq N}\left\{\lambda^{i}\right\}$ Clearly $L<\infty$ and $\lambda>0$ and for $x, y \in$ $K, q \in \mathcal{F}, t \geq 0$ and any $i \in\{1,2, \ldots, N\}$,

$$
\left\langle T_{i}(t) x-q, j(x-q)\right\rangle \leq\|x-q\|^{2}-\lambda\left\|x-T_{i}(t) x\right\|^{2}
$$

and

$$
\left\|T_{i}(t) x-T_{i}(t) y\right\| \leq L\|x-y\| .
$$

For a fixed $\delta \in(0,1)$ and $t \geq 0$ define a family $S_{i}(t): K \rightarrow K i=1,2, \ldots, N$ by

$$
S_{i}(t) x:=\left(1-\delta^{2}\right) x+\delta^{2} T_{i}(t) x, \quad \forall x \in K .
$$

Then, for $x, y \in K$ and $q \in \mathcal{F}$,

$$
\begin{aligned}
\left\langle S_{i}(t) x-q, j(x-q)\right\rangle & =\left(1-\delta^{2}\right)\langle x-q, j(x-q)\rangle+\delta^{2}\left\langle T_{i}(t) x-q, j(x-q)\right\rangle \\
& \leq\left(1-\delta^{2}\right)\|x-q\|^{2}+\delta^{2}\left[\|x-q\|^{2}-\lambda\left\|x-T_{i}(t) x\right\|^{2}\right] \\
& =\|x-q\|^{2}-\lambda \delta^{2}\left\|x-T_{i}(t) x\right\|^{2} .
\end{aligned}
$$

Let $\bar{\lambda}=\lambda \delta^{2}>0$, then

$$
\left\langle S_{i}(t) x-q, j(x-q)\right\rangle \leq\|x-q\|^{2}-\bar{\lambda}\left\|x-T_{i}(t) x\right\|^{2} .
$$

Also,

$$
\begin{aligned}
\left\|S_{i}(t) x-S_{i}(t) y\right\| & =\left\|\left(1-\delta^{2}\right)(x-y)+\delta^{2}\left(T_{i}(t) x-T_{i}(t) y\right)\right\| \\
& \leq\left(1-\delta^{2}\right)\|x-y\|+\delta^{2} L\|x-y\| \\
& =\left[1-\delta^{2}+\delta^{2} L\right]\|x-y\| \\
& \leq\left(1+\delta^{2} L\right)\|x-y\| .
\end{aligned}
$$

Let $\bar{L}=1+\delta^{2} L$. 
Then,

$$
\left\|S_{i}(t) x-S_{i}(t) y\right\| \leq \bar{L}\|x-y\| .
$$

Hence, for each $i \in\{1,2, \ldots N\}, S_{i}$ is Lipschitz with Lipschitz constant $\bar{L}>0$.

Lemma 3.1. Let $E$ be a real Banach space and $K$ be a nonempty closed convex subset of $E$. Let $\left\{\mathcal{J}_{i}\right\}_{i=1}^{N}$ be a finite family of Lipschitzian demi-contractive semigroups of $K$ with sequences of bounded measurable functions $L_{i}:[0, \infty) \rightarrow(0, \infty)$ and bounded functions $\lambda_{i}:[0, \infty) \rightarrow(0, \infty) i=1,2, \ldots, N$ such that for each $i=1,2, \ldots, N$,

$$
L^{i}:=\sup _{t \geq 0}\left\{L_{i}(t)\right\}<\infty, \lambda^{i}:=\inf _{t \geq 0}\left\{\lambda_{i}(t)\right\}>0 \text { and } F^{i}:=\bigcap_{t \geq 0} F\left(T_{i}(t)\right) \neq \emptyset .
$$

Let $\mathcal{F}:=\bigcap_{1 \leq i \leq N}\left\{\cap F\left(T_{i}(t)\right)\right\} \neq \emptyset,\left\{t_{n}\right\}$ be an increasing sequence in $[0, \infty)$ and $\left\{\alpha_{n}\right\}$ be a sequence in $(0,1)$ satisfying the following conditions:

(i) $\sum_{n=1}^{\infty}\left(1-\alpha_{n}\right)=\infty$, (ii) $\sum_{n=1}^{\infty}\left(1-\alpha_{n}\right)^{2}<\infty$.

Assume $\forall i \in\{1,2, \ldots, N\}$ for any bounded set $D \subset K$ the relation

$$
\lim _{n \rightarrow \infty} \sup _{x \in D, s \in \mathbb{R}^{+}}\left\|T_{i}\left(s+t_{n}\right) x-T_{i}\left(t_{n}\right) x\right\|=0
$$

holds. Let $\left\{x_{n}\right\}$ be a sequence generated by $x_{1} \in K$,

$$
x_{n+1}=\alpha_{n+1} x_{n}+\left(1-\alpha_{n+1}\right) S_{n+1}\left(t_{n+1}\right) x_{n}, n \geq 1
$$

where $T_{n}\left(t_{n}\right)=T_{n} \operatorname{modN}\left(t_{n}\right)$.

Then,

(a) $\lim _{n \rightarrow \infty}\left\|x_{n}-q\right\|$ lexists for all $q \in \mathcal{F}$.

(b) $\liminf _{n \rightarrow \infty}\left\|x_{n}-T_{i}\left(t_{n}\right) x_{n}\right\|=0$ for all $i \in\{1,2,3, \ldots, N\}$.

Proof. For any fixed $q \in \mathcal{F}$ using (3.5), we have

$$
x_{n+1}-q=\left(x_{n}-q\right)+\left(1-\alpha_{n+1}\right)\left(S_{n+1}\left(t_{n+1}\right) x_{n}-x_{n}\right) .
$$

Thus,

$$
\begin{aligned}
& \left\|x_{n+1}-q\right\|^{2}=\left\|\left(x_{n}-q\right)+\left(1-\alpha_{n+1}\right)\left(S_{n+1}\left(t_{n+1}\right) x_{n}-x_{n}\right)\right\|^{2} \\
& \quad \leq\left\|x_{n}-q\right\|^{2}+2\left(1-\alpha_{n+1}\right)\left\langle S_{n+1}\left(t_{n+1}\right) x_{n}-x_{n}, j\left(x_{n+1}-q\right)\right\rangle \\
& \quad=\left\|x_{n}-q\right\|^{2}+2\left(1-\alpha_{n+1}\right)\left[\left\langle S_{n+1}\left(t_{n+1}\right) x_{n}-S_{n+1}\left(t_{n+1}\right) x_{n+1}, j\left(x_{n+1}-q\right)\right\rangle\right. \\
& \quad+\left\langle S_{n+1}\left(t_{n+1}\right) x_{n+1}-q, j\left(x_{n+1}-q\right)\right\rangle-\left\langle x_{n+1}-q, j\left(x_{n+1}-q\right)\right\rangle \\
& \left.\quad+\left\langle x_{n+1}-x_{n}, j\left(x_{n+1}-q\right)\right\rangle\right] \\
& \quad \leq\left\|x_{n}-q\right\|^{2}+2\left(1-\alpha_{n+1}\right)(\bar{L}+1)\left\|x_{n}-x_{n+1}\right\| x_{n+1}-q \| \\
& \quad-2\left(1-\alpha_{n+1}\right) \bar{\lambda}\left\|x_{n+1}-T_{n+1}\left(t_{n+1}\right) x_{n+1}\right\|^{2} \\
& \quad \leq\left\|x_{n}-q\right\|^{2}+2\left(1-\alpha_{n+1}\right)^{2}(1+\bar{L})^{2}\left\|S_{n+1}\left(t_{n+1}\right) x_{n}-x_{n}\right\|\left\|x_{n}-q\right\| \\
& \quad-2\left(1-\alpha_{n+1}\right) \bar{\lambda}\left\|x_{n+1}-T_{n+1}\left(t_{n+1}\right) x_{n+1}\right\|^{2} \\
& \quad \leq\left\|x_{n}-q\right\|^{2}+2\left(1-\alpha_{n+1}\right)^{2}(1+\bar{L})^{3}\left\|x_{n}-q\right\|^{2} \\
& \quad-2\left(1-\alpha_{n+1}\right) \bar{\lambda}\left\|x_{n+1}-T_{n+1}\left(t_{n+1}\right) x_{n+1}\right\|^{2} \\
& \quad=\left(1+\sigma_{n+1}\right)\left\|x_{n}-q\right\|^{2}-2\left(1-\alpha_{n+1}\right) \bar{\lambda}\left\|x_{n+1}-T_{n+1}\left(t_{n+1}\right) x_{n+1}\right\|^{2} \\
& \leq\left(1+\sigma_{n+1}\right)\left\|x_{n}-q\right\|^{2},
\end{aligned}
$$


where $\sigma_{n+1}=2(1+\bar{L})^{3}\left(1-\alpha_{n+1}\right)^{2}$.

Since $\sum_{n=1}^{\infty}\left(1-\sigma_{n+1}\right)^{2}<\infty$, by lemma 2.2 , it follows that $\lim _{n \rightarrow \infty}\left\|x_{n}-q\right\|$ exists.

Hence, $\left\{x_{n}\right\}$ is bounded, which implies that $\left\{T_{n}\left(t_{n}\right) x_{n}\right\}$ and $\left\{S_{n}\left(t_{n}\right) x_{n}\right\}$ are also bounded.

From (3.6)

$$
\begin{aligned}
& \left\|x_{n+1}-q\right\|^{2} \leq\left\|x_{n}-q\right\|^{2}+2\left(1-\alpha_{n+1}\right)^{2}(1+\bar{L})^{3}\left\|x_{n}-q\right\|^{2} \\
& \quad-2\left(1-\alpha_{n+1}\right) \bar{\lambda}\left\|x_{n+1}-T_{n+1}\left(t_{n+1}\right) x_{n+1}\right\|^{2} \\
& \leq\left\|x_{n}-q\right\|^{2}-2\left(1-\alpha_{n+1}\right) \bar{\lambda}\left\|x_{n+1}-T_{n+1}\left(t_{n+1}\right) x_{n+1}\right\|^{2}+2\left(1-\alpha_{n+1}\right)^{2} M,
\end{aligned}
$$

where, $M:=(1+\bar{L})^{3} \sup _{n \in \mathbb{N}}\left(\left\|x_{n}-q\right\|^{2}\right)$. Hence, for some $m \in \mathbb{N}$,

$$
\begin{aligned}
2 \bar{\lambda} \sum_{n=1}^{m}\left(1-\alpha_{n+1}\right)\left\|x_{n+1}-T_{n+1}\left(t_{n+1}\right) x_{n+1}\right\|^{2} & \leq \sum_{n=1}^{m}\left(\left\|x_{n}-q\right\|^{2}-\left\|x_{n+1}-q\right\|^{2}\right) \\
& +2 M \sum_{n=1}^{m}\left(1-\alpha_{n+1}\right)^{2} \\
& \leq\left\|x_{1}-q\right\|^{2} \\
& +2 M \sum_{n=1}^{m}\left(1-\alpha_{n+1}\right)^{2}<\infty .
\end{aligned}
$$

Since $m \in \mathbb{N}$ is arbitrary, we have

$$
2 \bar{\lambda} \sum_{n=1}^{\infty}\left(1-\alpha_{n+1}\right)\left\|x_{n+1}-T_{n+1}\left(t_{n+1}\right) x_{n+1}\right\|^{2}<\infty
$$

which implies

$$
\liminf _{n \rightarrow \infty}\left\|x_{n+1}-T_{n+1}\left(t_{n+1}\right) x_{n+1}\right\|=0 .
$$

Next, we show that,

$$
\lim _{n \rightarrow \infty}\left\|x_{n+1}-x_{n}\right\|=0 .
$$

Let $\left\{\beta_{n}\right\}$ and $\left\{y_{n}\right\}$ be two sequences define by $\beta_{n}:=\delta(1-\delta) \alpha_{n+1}+\delta^{2}$ and $y_{n}:=\frac{x_{n+1}-x_{n}+\beta_{n} x_{n}}{\beta_{n}}$. Then, using the definition of $\left\{\beta_{n}\right\}$ and $\left\{S_{n}\right\}$ we obtain that $y_{n}:=\frac{\delta \alpha_{n+1} x_{n}+\delta^{2}\left(1-\alpha_{n+1}\right) T_{n+1}\left(t_{n+1}\right) x_{n}}{\beta_{n}}$. Then,

$$
\begin{aligned}
y_{n+1}-y_{n} & =\frac{\delta \alpha_{n+2}}{\beta_{n+1}}\left[x_{n+1}-x_{n}\right]+\delta\left[\frac{\alpha_{n+2}}{\beta_{n+1}}-\frac{\alpha_{n+1}}{\beta_{n}}\right] x_{n} \\
& +\frac{\delta^{2}\left(1-\alpha_{n+2}\right)}{\beta_{n+1}}\left[T_{n+2}\left(t_{n+2}\right) x_{n+1}-T_{n+2}\left(t_{n+2}\right) x_{n}\right] \\
& +\delta^{2}\left[\frac{1-\alpha_{n+2}}{\beta_{n+1}}-\frac{1-\alpha_{n+1}}{\beta_{n}}\right] T_{n+2}\left(t_{n+2}\right) x_{n} \\
& +\frac{\delta^{2}\left(1-\alpha_{n+1}\right)}{\beta_{n}}\left[T_{n+2}\left(t_{n+2}\right) x_{n}-T_{n+1}\left(t_{n+1}\right) x_{n}\right] .
\end{aligned}
$$


Therefore,

$$
\begin{aligned}
\left\|y_{n+1}-y_{n}\right\|-\left\|x_{n+1}-x_{n}\right\| & \leq\left(\frac{\delta \alpha_{n+2}}{\beta_{n+1}}+\frac{\delta^{2} L\left(1-\alpha_{n+2}\right)}{\beta_{n+1}}-1\right)\left\|x_{n+1}-x_{n}\right\| \\
& +\delta\left|\frac{\alpha_{n+2}}{\beta_{n+1}}-\frac{\alpha_{n+1}}{\beta_{n}}\right|\left\|x_{n}\right\| \\
& +\delta^{2}\left|\frac{1-\alpha_{n+2}}{\beta_{n+1}}-\frac{1-\alpha_{n+1}}{\beta_{n}}\right|\left\|T_{n+2}\left(t_{n+2}\right) x_{n}\right\| \\
& +\frac{\delta^{2}\left(1-\alpha_{n+1}\right)}{\beta_{n}}\left\|T_{n+2}\left(t_{n+2}\right) x_{n}-T_{n+1}\left(t_{n+1}\right) x_{n}\right\| .
\end{aligned}
$$

Hence,

$$
\limsup _{n \rightarrow \infty}\left(\left\|y_{n+1}-y_{n}\right\|-\left\|x_{n+1}-x_{n}\right\|\right) \leq 0,
$$

and by lemma 2.3,

$$
\lim _{n \rightarrow \infty}\left\|y_{n}-x_{n}\right\|=0 \text {. }
$$

Thus,

$$
\left\|x_{n+1}-x_{n}\right\|=\beta_{n}\left\|y_{n}-x_{n}\right\| \rightarrow 0 \text { as } n \rightarrow \infty .
$$

This implies that,

$$
\left\|x_{n+i}-x_{n}\right\| \rightarrow 0 \text { as } n \rightarrow \infty, \forall i \in\{1,2,3, \ldots, N\} .
$$

But, for $i \in\{1,2,3, \ldots, N\}$,

$$
\begin{aligned}
\left\|x_{n}-S_{n+i}\left(t_{n+i}\right) x_{n}\right\| & \leq \delta^{2}\left[\left\|x_{n}-x_{n+i}\right\|+\left\|x_{n+i}-T_{n+i}\left(t_{n+i}\right) x_{n+i}\right\|\right. \\
& \left.+\left\|T_{n+i}\left(t_{n+i}\right) x_{n+i}-T_{n+i}\left(t_{n+i}\right) x_{n}\right\|\right] \\
& \leq \delta^{2}\left[(1+L)\left\|x_{n+i}-x_{n}\right\|+\left\|x_{n+i}-T_{n+i}\left(t_{n+i}\right) x_{n+i}\right\|\right] .
\end{aligned}
$$

Therefore,

$$
\liminf _{n \rightarrow \infty}\left\|x_{n}-S_{n+i}\left(t_{n+i}\right) x_{n}\right\|=0 .
$$

Hence,

$$
\liminf _{n \rightarrow \infty}\left\|T_{n+i}\left(t_{n+i}\right) x_{n}-x_{n}\right\|=\liminf _{n \rightarrow \infty}\left[\frac{1}{\delta^{2}}\left\|S_{n+i}\left(t_{n+i}\right) x_{n}-x_{n}\right\|\right]=0 .
$$

From the relation,

$$
\begin{aligned}
& \left\|T_{n+i}\left(t_{n}\right) x_{n}-x_{n}\right\| \leq\left\|T_{n+i}\left(t_{n}\right) x_{n}-T_{n+i}\left(\left(t_{n+i}-t_{n}\right)+t_{n}\right) x_{n}\right\| \\
& \quad+\left\|T_{n+i}\left(t_{n+i}\right) x_{n}-x_{n}\right\| \\
& \leq \sup _{z \in\left\{x_{n}\right\}, s \in \mathbb{R}^{+}}\left\|T_{n+i}\left(t_{n}\right) z-T_{n+i}\left(s+t_{n}\right) z\right\|+\left\|T_{n+i}\left(t_{n+i}\right) x_{n}-x_{n}\right\|,
\end{aligned}
$$

and condition (3.4) we get

$$
\liminf _{n \rightarrow \infty}\left\|T_{n+i}\left(t_{n}\right) x_{n}-x_{n}\right\|=0 .
$$

It follows from (3.8) that $\liminf _{n \rightarrow \infty}\left\|T_{l}\left(t_{n}\right) x_{n}-x_{n}\right\|=0 \forall l \in\{1,2,3, \ldots, N\}$. This completes the proof. $\quad$ - 
Theorem 3.2. Let $E, K, \mathcal{F},\left\{\alpha_{n}\right\},\left\{t_{n}\right\},\left\{\mathcal{J}_{i}\right\}_{i=1}^{N}$ and $\left\{x_{n}\right\}$ be as in lemma 3.1. Assume that, for at least one $i \in\{1,2, \ldots, N\}$, there exists a compact subset $C$ of $E$ such that $\mathrm{U}_{t \geq 0} T_{i}(t)$ $(K) \subset C$. Then, the sequence $\left\{x_{n}\right\}$ converges to some element $\mathcal{F}$.

Proof. By Lemma 3.1, we have $\liminf _{n \rightarrow \infty}\left\|T_{l}\left(t_{n}\right) x_{n}-x_{n}\right\|=0 \forall l \in\{1,2,3, \ldots, N\}$.

If $\mathrm{U}_{t \geq 0} T_{s}(t)(K) \subset C$ for some compact subet $C$ of $E$ and some $s \in\{1,2, \ldots, N\}$, then there exists a subsequence $\left\{x_{n_{k}}\right\}$, of $\left\{x_{n}\right\}$ and $q^{*} \in K$, such that

$$
x_{n_{k}} \rightarrow q^{*} \text { and }\left\|T_{s}\left(t_{n_{k}}\right) x_{n_{k}}-x_{n_{k}}\right\| \rightarrow 0 \text { as } n \rightarrow \infty .
$$

Observe that for $t>0$,

$$
\begin{aligned}
\left\|T_{s}(t) x_{n_{k}}-x_{n_{k}}\right\| & \leq\left\|T_{s}(t) x_{n_{k}}-T_{s}(t) T_{s}\left(t_{n_{k}}\right) x_{n_{k}}\right\| \\
& +\left\|T_{s}(t) T_{s}\left(t_{n_{k}}\right) x_{n_{k}}-T_{s}\left(t_{n_{k}}\right) x_{n_{k}}\right\|+\left\|T_{s}\left(t_{n_{k}}\right) x_{n_{k}}-x_{n_{k}}\right\| \\
& \leq\left\|T_{s}\left(t+t_{n_{k}}\right) x_{n_{k}}-T_{s}\left(t_{n_{k}}\right) x_{n_{k}}\right\|+(1+L)\left\|T_{s}\left(t_{n_{k}}\right) x_{n_{k}}-x_{n_{k}}\right\| .
\end{aligned}
$$

From the above we have $\lim _{k \rightarrow \infty}\left\|T_{s}(t) x_{n_{k}}-x_{n_{k}}\right\|=0$. Using (3.9) and the fact that $T s$ is Lipschitzian, we get $q^{*} \in \cap_{t \geq 0} F\left(T_{s}(t)\right)$.

Now, for any $l \in\{1,2, \ldots, N\}$, since $\liminf _{k \rightarrow \infty}\left\|T_{l}\left(t_{n_{k}}\right) x_{n_{k}}-x_{n_{k}}\right\|=0$, there exists a subsequence $\left\{x_{n_{k_{j}}}\right\}$ of $\left\{x_{n_{k}}\right\}$ such that

$\lim _{j \rightarrow \infty}\left\|T_{l}\left(t_{n_{k_{j}}}\right) x_{n_{k_{j}}}-x_{n_{k_{j}}}\right\|=\liminf _{k \rightarrow \infty}\left\|T_{l}\left(t_{n_{k}}\right) x_{n_{k}}-x_{n_{k}}\right\|=0$. Then, similarly for $t \geq 0$, we obtain

$$
\begin{aligned}
& \left\|T_{l}(t) x_{n_{k_{j}}}-x_{n_{k_{j}}}\right\| \leq\left\|T_{l}(t) x_{n_{k_{j}}}-T_{l}(t) T_{l}\left(t_{n_{k_{j}}}\right) x_{n_{k_{j}}}\right\| \\
& \quad+\left\|T_{l}(t) T_{l}\left(t_{n_{k_{j}}}\right) x_{n_{k_{j}}}-T_{l}\left(t_{n_{k_{j}}}\right) x_{n_{k_{j}}}\right\|+\left\|T_{l}\left(t_{n_{k_{j}}}\right) x_{n_{k_{j}}}-x_{n_{k_{j}}}\right\| \\
& \quad \leq\left\|T_{l}\left(t+t_{n_{k_{j}}}\right) x_{n_{k_{j}}}-T_{l}\left(t_{n_{k_{j}}}\right) x_{n_{k_{j}}}\right\|+(1+L)\left\|T_{l}\left(t_{n_{k_{j}}}\right) x_{n_{k_{j}}}-x_{n_{k_{j}}}\right\| .
\end{aligned}
$$

This implies that $\lim _{j \rightarrow \infty}\left\|T_{l}(t) x_{n_{k_{j}}}-x_{n_{k_{j}}}\right\|=0$ and hence $q^{*} \in \cap_{t \geq 0} F\left(T_{l}(t)\right)$. Since $l \in\{1$, $2, \ldots N\}$ is arbitrarily chosen, we have $q^{*} \in \mathcal{F}$. As the limit $\lim _{n \rightarrow \infty}\left\|x_{n}-q^{*}\right\|$ exists, the conclusion of the theorem follows immediately and this completes the proof.

Remark 3.3. Observe that considering a single one-parameter family of demi-contractive semigroup in Theorem 3.2, we obtain the conclusion of Theorem 1.1.

Let $T_{1}, T_{2}, \ldots, T_{N}$ be a finite family of Lipschitzian demi-contractive self-mapping of $K$ with positive constants $\lambda_{1}, \lambda_{2}, \ldots, \lambda_{N}$ and Lipschitz constants $L_{1}, L_{2}, \ldots, L_{N}$,

respectively. Let $F:=\bigcap_{1 \leq i \leq N} F\left(T_{i}\right) \neq \emptyset$.

For a fixed $\delta \in(0,1)$, define $S_{n}: K \rightarrow K$ by

$$
S_{n} x:=\left(1-\delta^{2}\right) x+\delta^{2} T_{n} x, \quad \forall x \in K .
$$

Then, it follows that for $x, y \in K$ and $i \in F$,

$$
\begin{gathered}
\left\langle S_{n} x-q, j(x-q)\right\rangle \leq\|x-q\|^{2}-\bar{\lambda}\left\|x-T_{n} x\right\|^{2} \text { and } \\
\left\|S_{n} x-S_{n} y\right\| \leq \bar{L}\|x-y\|,
\end{gathered}
$$

where $\bar{\lambda}=\lambda \delta^{2}>0, \bar{L}=1+\delta^{2} L, \lambda:=\min _{1 \leq i \leq N}\left\{\lambda_{i}\right\}$ and $L:=\max _{1 \leq i \leq N}\left\{L_{i}\right\}$.

The following Theorem is a consequence of Lemma 3.1.

Theorem 3.4. Let $E, K$ and $\left\{\alpha_{n}\right\}$ be as in Lemma 3.1. Let $T_{1}, T_{2}, \ldots, T_{N}: K \rightarrow K$ be Lipschitzian demi-contractive mappings with $T_{s}$ demicompact for at least one $s \in\{1,2$, 
..., N\}. Let $\left\{x_{n}\right]$ be a sequence generated by $x_{1} \in K$

$$
x_{n+1}=\alpha_{n+1} x_{n}+\left(1-\alpha_{n+1}\right) S_{n+1} x_{n},
$$

where $T_{n}=T_{n} \operatorname{modN}$. Then, $\left\{x_{n}\right\}$ converges strongly to a common fixed point of the family $\left\{T_{i}\right\}_{i=1}^{N}$.

Proof. Following the line of proof of lemma 3.1 we immediately obtain $\lim _{n \rightarrow \infty}\left\|x_{n}-q\right\| q k$ exists for any $q \in F$ and $\liminf _{n \rightarrow \infty}\left\|T_{i} x_{n}-x_{n}\right\|=0, \forall i \in\{1,2, \ldots N\}$. Let $\left\{x_{n_{k}}\right\}$ be a subsequence of $\left\{x_{n}\right\}$ such that

$$
\lim _{k \rightarrow \infty}\left\|T_{i} x_{n_{k}}-x_{n_{k}}\right\|=\liminf _{n \rightarrow \infty}\left\|T_{i} x_{n}-x_{n}\right\|=0 .
$$

Therefore $\lim _{k \rightarrow \infty}\left\|T_{s} x_{n_{k}}-x_{n_{k}}\right\|=0$ and, by demicompactness of $T_{s}$, there exists a subsequence $\left\{x_{n_{k_{j}}}\right\}$ of $\left\{x_{n_{k}}\right\}$ and $q^{*} \in K$, such that $x_{n_{k_{j}}} \rightarrow q^{*}$ as $j \rightarrow \infty$.

Since,

$$
\begin{aligned}
0=\lim _{j \rightarrow \infty}\left\|T_{i} x_{n_{k_{j}}}-x_{n_{k_{j}}}\right\| & =\left\|T_{i} \lim _{j \rightarrow \infty} x_{n_{k_{j}}}-\lim _{j \rightarrow \infty} x_{n_{k_{j}}}\right\| \\
& =\left\|T_{i} q^{*}-q^{*}\right\|,
\end{aligned}
$$

we obtain $q^{*} \in F$. But, $\lim _{n \rightarrow \infty}\left\|x_{n}-q^{*}\right\|$ exists, thus $x_{n} \rightarrow q^{*} \in F$ and this completes the proof.

The following corollaries follow from Theorem 3.4

Corollary 3.5. Let $E, K$ and $\left\{\alpha_{n}\right\}$ be as in Theorem 3.4. Let $T_{1}, T_{2}, \ldots, T_{N}: K \rightarrow K$ be Lipschitzian demi-contractive mappings. Suppose there exists a compact subset $C$ in $E$ such that $\bigcup_{i=1}^{N} T_{i}(K) \subset C$. Let $\left\{x_{n}\right\}$ be defined by (3.11). Then, $\left\{x_{n}\right\}$ converges strongly to a common fixed point of the family $\left\{T_{i}\right\}_{i=1}^{N}$.

Corollary 3.6. Let $E ; K$ and $\left\{\alpha_{n}\right\}$ be as in Theorem 3.4. Let $T_{1}, T_{2}, \ldots, T_{N}: K \rightarrow K$ be Lipschitzian demi-contractive mappings satisfying condition $\bar{C}$. Let $\left\{x_{n}\right\}$ be defined by (3.11). Then, $\left\{x_{n}\right\}$ converges strongly to a common fixed point of the family $\left\{T_{i}\right\}_{i=1}^{N}$.

Proof. Following the line of proof of lemma 3.1, we obtain $\liminf _{n \rightarrow \infty}\left\|x_{n}-T_{i} x_{n}\right\|=0$ for all $i \in\{1,2,3, \ldots, N\}$ and $\left\|x_{n+1}-q\right\|^{2} \leq\left(1+\sigma_{n+1}\right)\left\|x_{n}-q\right\|^{2}$, where $\sigma_{n+1}=2(1+\bar{L})^{3}\left(1-\alpha_{n+1}\right)^{2}$. Since $\sum_{n=1}^{\infty}\left(1-\sigma_{n+1}\right)^{2}<\infty$, by lemma $2.2 \lim _{n \rightarrow \infty}\left\|x_{n}-p\right\|$ exists and consequently $\lim _{n \rightarrow \infty} d\left(x_{n}, F\right)$ exists. Let $\left\{x_{n_{k}}\right\}$ be a subsequence of $\left\{x_{n}\right\}$ such that $\lim _{k \rightarrow \infty}\left\|x_{n_{k}}-T_{i} x_{n_{k}}\right\|=\liminf _{n \rightarrow \infty}\left\|x_{n}-T_{i} x_{n}\right\|=0$. Then, by using condition $\bar{C}$, there exists $s \in\{1,2, \ldots, N\}$ such that $0=\lim _{k \rightarrow \infty}\left\|x_{n_{k}}-T_{s} x_{n_{k}}\right\| \geq \lim _{k \rightarrow \infty} f\left(d\left(x_{n_{k}}, F\right)\right)$ and, using the property of $f$, we get that $\lim _{k \rightarrow \infty} d\left(x_{n_{k}}, F\right)=0$, and since the limit $\lim _{n \rightarrow \infty} d\left(x_{n}, F\right)$ exists we have that $\lim _{n \rightarrow \infty} d\left(x_{n}, F\right)=0$. We next show that $\left\{x_{n}\right\}$ is Cauchy. Let $\varepsilon>0$ be given, then there exists $p^{*} \in F$ and $n^{*} \in \mathbb{N}$ such that $\forall n \geq n^{*},\left\|x_{n}-p^{*}\right\|<\frac{\varepsilon}{2}$. Hence, for $n \geq$ $n^{*}$ and $k \in \mathbb{N}$, we have

$$
\begin{aligned}
\left\|x_{n+k}-x_{n}\right\| & \leq\left\|x_{n+k}-p^{*}\right\|+\left\|x_{n}-p^{*}\right\| \\
& <\varepsilon .
\end{aligned}
$$

Thus, $\left\{x_{n}\right\}$ is Cauchy and so $x_{n} \rightarrow q^{*} \in \mathrm{K}$. We now show that $q^{*}$ is in F. Since $\lim _{n \rightarrow \infty} d\left(x_{n}, F\right)=0$, there exists $m_{0} \in \mathbb{N}$ large enough and $p^{*} \in F$ such that for all $n \geq m_{0}$, 


$$
\begin{aligned}
& \text { and }\left\|x_{n}-p^{*}\right\|<\frac{\varepsilon}{6(1+L)} \text {. Hence, } \\
& \qquad \begin{aligned}
\left\|q^{*}-T_{l} q^{*}\right\| & \leq\left\|x_{n}-q^{*}\right\|+\left\|x_{n}-p^{*}\right\|+\left\|p^{*}-T_{l} q^{*}\right\| \\
& \leq \frac{\varepsilon}{6(1+L)}+\frac{\varepsilon}{6(1+L)}+L\left\|p^{*}-q^{*}\right\| \\
& <\frac{\varepsilon}{6(1+L)}+\frac{\varepsilon}{6(1+L)}+\frac{3 L \varepsilon}{6(1+L)} \\
& <\varepsilon .
\end{aligned}
\end{aligned}
$$

Thus, $q^{*} \in F\left(T_{l}\right)$ and since $l \in\{1,2, \ldots, N\}$ is arbitrary, we have $q^{* *} \in F$. This completes the proof.

\section{Acknowledgements}

This study was conducted when the first author was visiting the AbdusSalam International Center for Theoretical Physics Trieste Italy as an Associate, and the hospitality and financial support provided by the centre is gratefully acknowledged.

\section{Authors' contributions}

BA conceived the study, GCU carried out the computations for Theorem 3.4. BA Modified Theorem 3.4 to obtain Theorem 3.2. Both authors read and approved the final manuscript.

\section{Competing interests}

The authors declare that they have no competing interests.

Received: 6 March 2011 Accepted: 23 July 2011 Published: 23 July 2011

\section{References}

1. Belluce, LP, Kirk, WA: Fixed point theorem for families of contraction mappings. Pacific J Math. 18, 213-217 (1966)

2. Browder, FE: Nonexpansive nonlinear operators in Banach space. Proc Natl Acad Sci USA. 54, 1041-1044 (1965). doi:10.1073/pnas.54.4.1041

3. Bruck, RE: A common fixed point theorem for a commuting family of nonexpansive mappings. Pacific J Math. 53 , 59-71 (1974)

4. De Marr, R: Common fixed points for commuting contraction mappings. Pacific J Math. 13, 1139-1141 (1963)

5. Lim, TC: A fixed point theorem for families of nonexpansive mappings. Pacific J Math. 53, 487-493 (1974)

6. Suzuki, T: Fixed point property for nonexpansive mappings versus that for nonexpansive semigroups. Nonlinear Anal. 70, 3358-3361 (2009). doi:10.1016/j.na.2008.05.003

7. Shoiji, N, Takahashi, W: Strong convergence theorem for asymptotically nonexpansive semi-groups in Hilbert spaces. Nonlinear Anal. 34, 87-99 (1998). doi:10.1016/50362-546X(97)00682-2

8. Suzuki, T: On strong convergence to a common fixed point of nonexpansive semigroups in Hilbert spaces. Proc Am Math Soc. 131, 2133-2136 (2003). doi:10.1090/50002-9939-02-06844-2

9. $\mathrm{Xu}, \mathrm{HK}$ : Strong convergence theorem for contraction semigroups in Banach spaces. Bull Austal Math Soc. 72, 371-379 (2005). doi:10.1017/S000497270003519X

10. Aleyner, A, Reich, S: An explicit construction of sunny nonexpansive retraction in Banach spaces. Fixed Point Theory Appl. 3, 295-305 (2005)

11. Zhang, SS, Yang, L, Liu, JA: Strong convergence theorem for nonexpansive semigroups in Banach spaces. Appl Math Mech. 28, 1287-1297 (2007). doi:10.1007/s10483-007-1002-x

12. Chang, SS, Cho, YJ, Lee, HWJ, Chan, C: Strong convergence theorems for Lipschitzian demicontraction semigroups in Banach spaces, Fixed Point Theory Application. (2011)

13. Zhang, SS: Convergence theorem of common fixed points for Lipshitzian pseudocontraction semigroups in Banach spaces. Appl Math Mech. 30, 145-152 (2009). doi:10.1007/s10483-009-0202-y

14. Xu, HK: Inequalities in Banach spaces with applications. Nonlinear Anal. 16, 1127-1138 (1991). doi:10.1016/0362-546X(91) 90200-K

15. Suzuki, T: Strong convergence of Krasnoselskii and Mann's type sequences for one-parameter nonexpansive semigroups without Bochner integrals. J Math Anal Appl. 305, 227-239 (2005). doi:10.1016/j.jmaa.2004.11.017

doi:10.1186/1687-1812-2011-18

Cite this article as: Ali and Ugwunnadi: Convergence theorem for finite family of lipschitzian demi-contractive

semigroups. Fixed Point Theory and Applications 2011 2011:18. 